Volume 8. No. 1.1, 2020

International Journal of Emerging Trends in Engineering Research

Available Online at http://www.warse.org/IJETER/static/pdf/file/ijeter1081.12020.pdf

https://doi.org/10.30534/ijeter/2020/1081.12020

\title{
Optimal Placement of Distributed Generation in Transmission Network for Loss Minimization via Multistage Artificial Immune System
}

\author{
Nurzanariah Roslan ${ }^{1}$, Mohd Helmi Mansor ${ }^{2}$, Wan Muhammad Mirza Wan Azahari ${ }^{3}$, Ismail Musirin ${ }^{4}$, \\ Sharifah AzwaShaaya ${ }^{5}$ \\ 1,2,3,5 Department of Electrical and Electronics Engineering, College of Engineering, Universiti Tenaga Nasional, \\ Selangor, Malaysia, ${ }^{1}$ nurzanariah@ uniten.edu.my, ${ }^{2}$ mhelmi@uniten.edu.my, \\ 3mijra.16@gmail.com, ${ }^{5}$ shazwa@ uniten.edu.my \\ ${ }^{4}$ Faculty of Electrical Engineering, UniversitiTeknologi MARA, Selangor, Malaysia, ${ }^{4}$ ismailbm1@ gmail.com
}

\begin{abstract}
This paper presents the application of a modified Artificial Immune System (AIS) technique to determine the optimal location and sizing of Distributed Generation (DG) in transmission network. The technique is termed as Multistage Artificial Immune System (MAIS). The IEEE 26-Bus Reliability Test System (RTS) has been utilized to conduct the implementation. Prior to the MAIS deployment, the original Artificial Immune System (AIS) technique has been used to find the optimal placement of the DG units in the IEEE 26-Bus RTS. This is for comparative study purposes. Three case studies with different conditions of transmission network have been introduced in finding the optimal location and sizing of the DG units. It is found that MAIS is superior than the original AIS in finding the optimal placement of the DG units in the transmission network in terms of producing low total system loss.
\end{abstract}

Key words : Distributed Generation, Optimal Placement, Multistage Artificial Immune System.

\section{INTRODUCTION}

Distributed Generation (DG) technologies have been growing significantly in the electricity utility system around the world for the past two decades. The growth of DG technologies around the world are mainly due to numerous factors and drivers. DG is often used to improve reliability or to delay investment in transmission and distribution networks, avoid network charges, reduce line losses, stop the construction of large generation installations, displace expensive grid-supplied energy, provide alternative sources of market supply and provide environmental benefits. As compared to a typical large centralized power plant, DG is much more superior in terms of cost effectiveness and efficiency. There are two main technological sources that drives the power generation of a DG which are Fossil fuel based and
Non-Fossil fuel based. This basically means that the sources used to generate power in the plant can either be from a renewable source or a non-renewable source such a coal, natural gas, solar, wind or hydro which will be discussed further in the report.

There are many advantages to have DG in the transmission network. DG able to directly provide supply to consumers in a certain location where DG power plant is built. In a certain area where the consumer load is far away from the transmission lines, the existence of DG connected to the grid nearby the consumer load can directly provide power generation and electricity to the consumer load. This in terms means that DGs can reduce the consumer total load and hence reduce the loading on the power transmission lines. In the event of an emergency where the breakdown occurs at power transmission lines or in distribution network, DGs are able to provide supply to some areas to reduce the number of power outages in the areas [1]. When an outage occurs in the transmission lines the distribution of power in the lines become imbalance. Hence the DGs may provide an injection of power to balance the power flow in the transmission network and therefore reduce the total power loss in the system. Besides that, through the control of reactive power and active power of the DG technologies the voltage variance in the system can be further reduced to supply accurate voltage level. Reactive power plays an important role in reducing the losses whereby it improves the power factor in the transmission network therefore reducing the amount of real power needed to supply the load on the consumer load. Hence the losses in the system can also be reduced and thus enhance the efficiency in the system supply. Moreover, the construction of DG power plant is much faster compared to the centralized power plant due to the smaller size and rather simple system to be installed. The growth of load demand is higher than production of electricity, so the installation of DG is crucial to support the network in short duration. Storms, falling tree branches, brownouts, and acts of terror challenge the grid and when it fails, it usually leaves tens of thousands of 
customers (or millions in extreme cases) without electricity for long periods of time. The effect of these failures can be detected by a distributed microgrid generation system, which decreases the number of people affected [1].

The integration of DGs into transmission network involves a wide range of techniques that were made by engineers and researchers worldwide. Some of the techniques are Genetic Algorithm (GA) [2], Tabu Search [3], Ant Colony Optimization (ACO) [4], Artificial Bee Colony (ABC) [5], Harmony Search Algorithm (HSA) [6], Evolutionary Programming (EP) [7] and Artificial Immune System (AIS) [8]. These techniques use specific algorithm programmed by the designers that can enhance the efficiency in the transmission network by reducing the system losses, improve voltage stability, reduce operational cost and other important elements. Among the techniques, AIS and EP are commonly used to solve transmission network optimization problems including the optimal DG placement. However, sometimes EP and AIS cannot provide optimal solution because of the randomization of generating the control variables' values during initialization process that produced unfit individuals. Furthermore, there is possibility for the optimization process to get stuck at its local optima if the starting point of the search is not properly set or the objective function of the problem is non-convex [9], [10].

In this paper, an artificial intelligence technique termed as Multistage Artificial Immune System (MAIS) is proposed to be used to determine the optimal location and sizing of Distributed Generation (DG) in the transmission network. The optimization results produced by MAIS has been compared with the optimization results produced by the original AIS. It is found that, MAIS produced the lowest total system loss compared to AIS for several conditions of transmission network.

\section{METHODOLOGY}

Unlike AIS, MAIS has multiple stages in its processes. After the initialization process, the generated control variables values are decomposed into several stages depending on the size of the initial population. The control variables for this study is the location and sizing of the DG units. The number of individuals in each stage is equally divided. For example, if the size of initial population is twenty, there will be five individuals in each stage if there are four stages. Subsequently, the five individuals in each stage will undergo cloning process, mutation process and selection process. During cloning process, the individuals generated during the initialization are multiplied by ten to become fifty individuals. While during mutation, the cloned individuals are mutated using Gaussian mutation to produce offspring. The Gaussian mutation is as shown in equation 1.

$$
x_{i+m, j}=x_{i, j}+N\left(0, \beta\left(x_{\text {jmax }}-x_{\text {jmin }}\right)\left(\frac{f_{i}}{f_{\text {max }}}\right)\right)
$$

Where:

$$
\begin{array}{ll}
x_{i+m, j} & =\text { mutated parent (offspring) } \\
x_{i, j} & =\text { parents } \\
N & =\text { Gaussian random variable with mean } \mu \text { and } \\
\text { variance } y^{2} & \\
\beta \quad=\text { mutation scale, } 0<\beta<1 \\
x_{\text {jmax }} \quad=\text { maximum random number for every variable } \\
x_{\text {jmin }} \quad=\text { minimum random number for every variable } \\
f_{i} \quad=\text { fitness for the } i^{t h} \text { random number } \\
f_{\text {max }} \quad=\text { maximum fitness }
\end{array}
$$

During selection process, the mutated individuals are ranked based on the best fitness of total system loss and then five fittest individuals are selected for the next process. After the selection process, the fittest individuals from each stage are combined to become a new big population. Then, the new population will undergo convergence test where the global optima will be found if it meets the set stopping criterion. The flowchart of MAIS for optimal placement of DG in transmission network is illustrated in Figure 1.

For this study, five DG units are used to be optimally placed in the IEEE 26-Bus RTS using MAIS. These DG units are Type I, which can only supply active power to the system. The objective function of this optimization problem is to minimize total system loss, which can be written as follows:

$$
\begin{gathered}
P_{\text {loss }}=\sum_{k=1}^{l} g_{k}\left[V_{i}^{2}+V_{j}^{2}-2 V_{i} V_{j} \cos \left(\delta_{i}-\delta_{j}\right),\right. \\
k \in\{1,2, \ldots, l\}
\end{gathered}
$$

Where,

$g_{k}$ is conductance of $k^{\text {th }}$ line,

$V_{i}$ and $\delta_{i}$ are voltage magnitude and angle of bus $i$, respectively,

$V_{j}$ and $\delta_{j}$ are voltage magnitude and angle of bus $j$, respectively, and

$l$ is number of lines in the system 


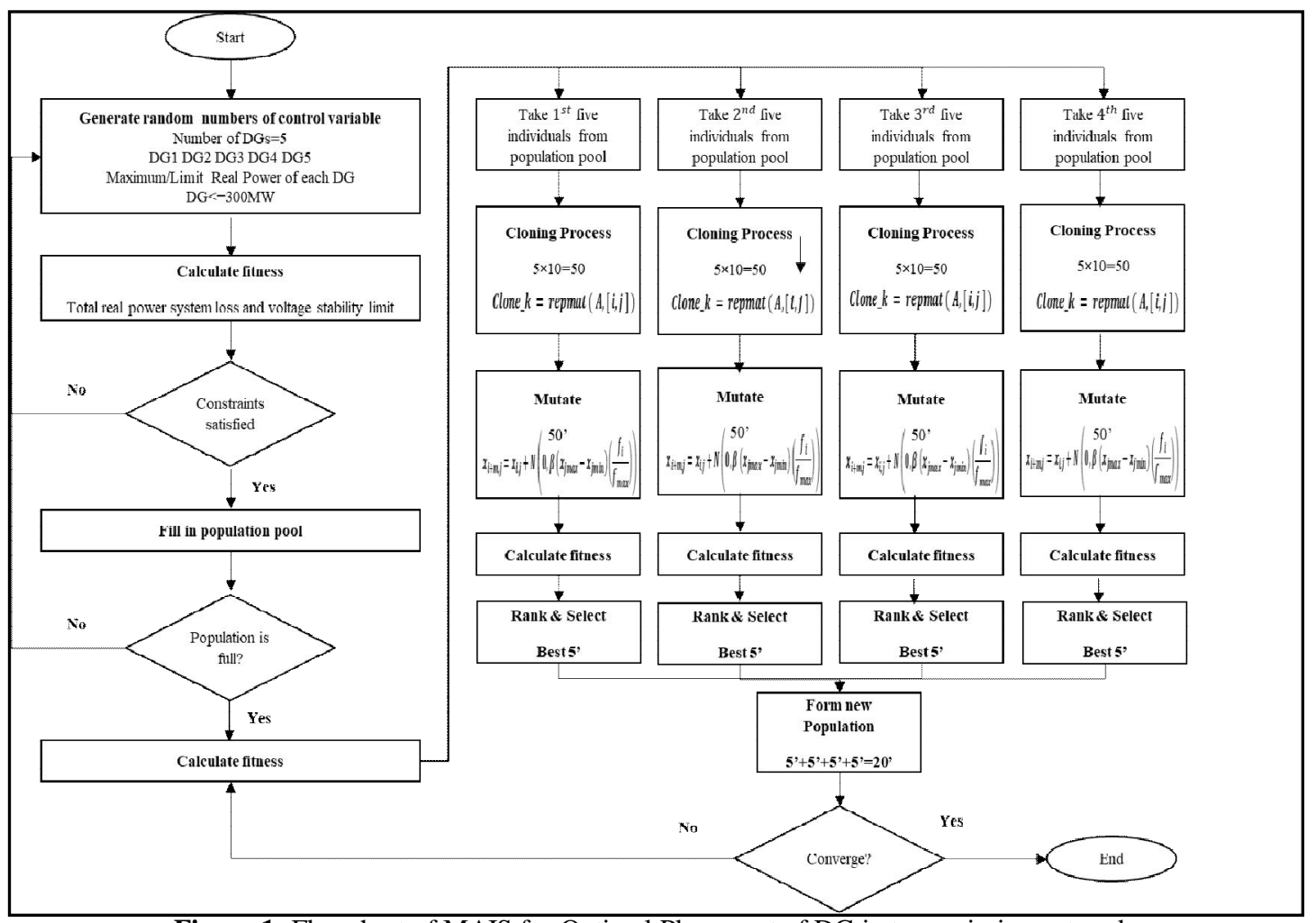

Figure 1: Flowchart of MAIS for Optimal Placement of DG in transmission network

\section{RESULTS AND DISCUSSION}

The developed MAIS program was used to solve optimal distributed generation installation problem with three different case studies. This is important to study the performance of the proposed technique in finding the best location and size of each DG unit in the transmission network by having different conditions of system. For the first case study, MAIS program was used to solve base case in which the transmission network is not modified. In the second case study, (N-1) line contingency analysis was performed, which one line was put on outage. The effect of the outage on the final solution was monitored by observing the total system loss produced. Lastly, (N-2) line contingency analysis was carried out whereby two lines in the system were put on outage. This process was done by removing two lines in the IEEE 26-Bus RTS and MAIS program was deployed to find the best location and size of each DG unit. The results produced by MAIS were compared with the results produced using AIS for all the three case studies.

\subsection{Case study 1: Base case}

Figure 2 shows the comparison of total system losses found by MAIS and AIS for the base case. Based on Figure 2, it can be observed that there is a reduction in the average loss between the AIS and MAIS. The approximate reduction in the average loss from AIS to MAIS is about $7.39 \%$. It can also be seen that the best loss for MAIS is lower as compared to AIS by about $7.74 \%$. The worst loss is also reduced using MAIS by about $6.66 \%$ as compared to the AIS. From the results, it can be concluded that MAIS significantly reduced the total power loss in the system for the base case where there is no modification made on the IEEE 26-Bus RTS. The reason for the decrement in the total system loss is due to the wider range of possible solutions created by MAIS as compared to AIS.

\subsection{Case Study 2: (N-1) Line Contingency}

Based on Figure 3, the worst loss found by MAIS is seen to be higher than AIS, which are 14.6972 MW and 14.3953 MW, respectively. The percentage difference of the losses between the two techniques is about a surplus of $1.97 \%$. However, MAIS managed to obtain a lower best loss as compared to AIS. The best loss for MAIS throughout the 10 runs is at 13.1275 MW which is $1.78 \%$ less than the best loss of AIS. Moreover, the average loss for all the 10 runs done by MAIS is also lower than AIS by $0.14 \%$. It is expected to see the increase in the total power loss in the system when an outage occurs in the transmission line for both MAIS and AIS program. However, the MAIS managed to obtain a better reduction in loss as compared to AIS. 
Nurzanariah Roslan et al., International Journal of Emerging Trends in Engineering Research, 8(1.1), 2020, 68 - 72

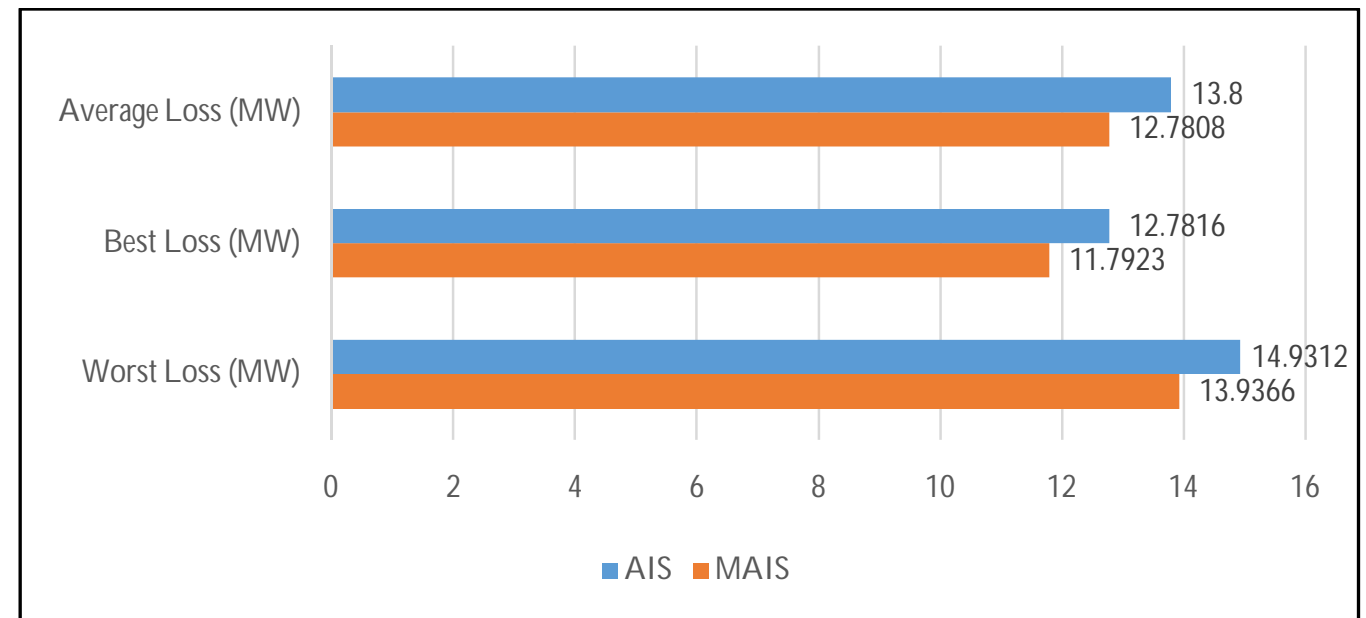

Figure 2: Average Loss, Best Loss and Worst Loss for MAIS and AIS (Base Case)

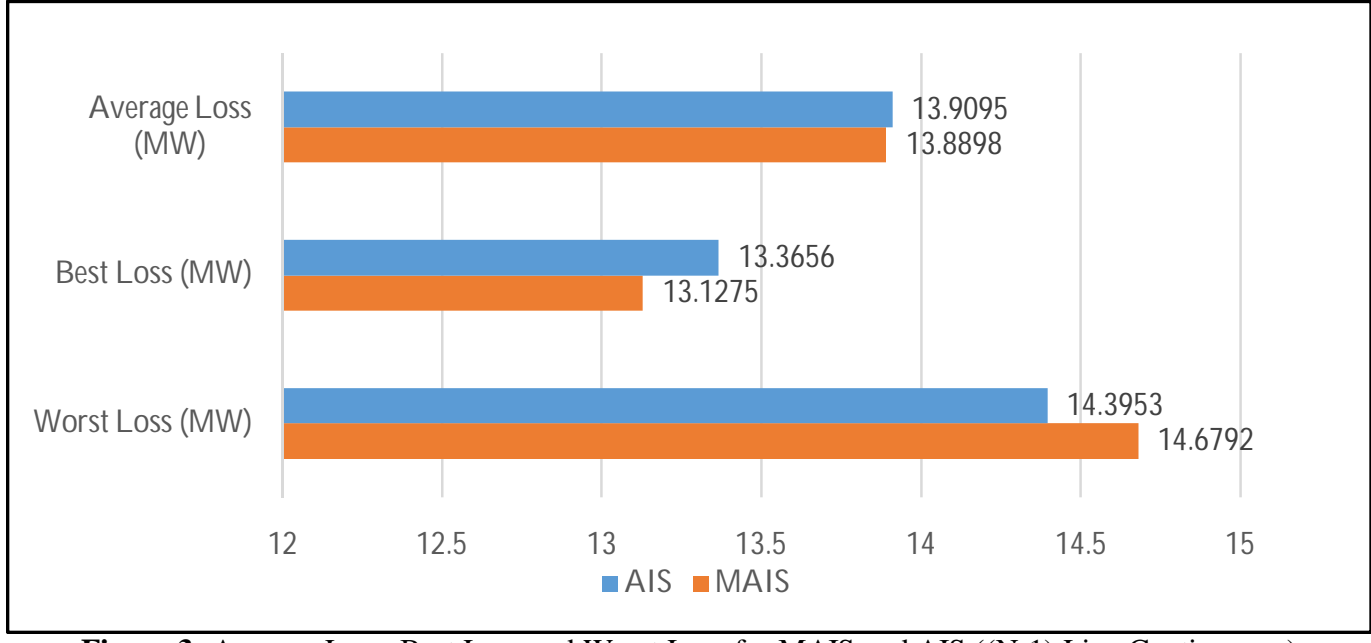

Figure 3: Average Loss, Best Loss and Worst Loss for MAIS and AIS ((N-1) Line Contingency)

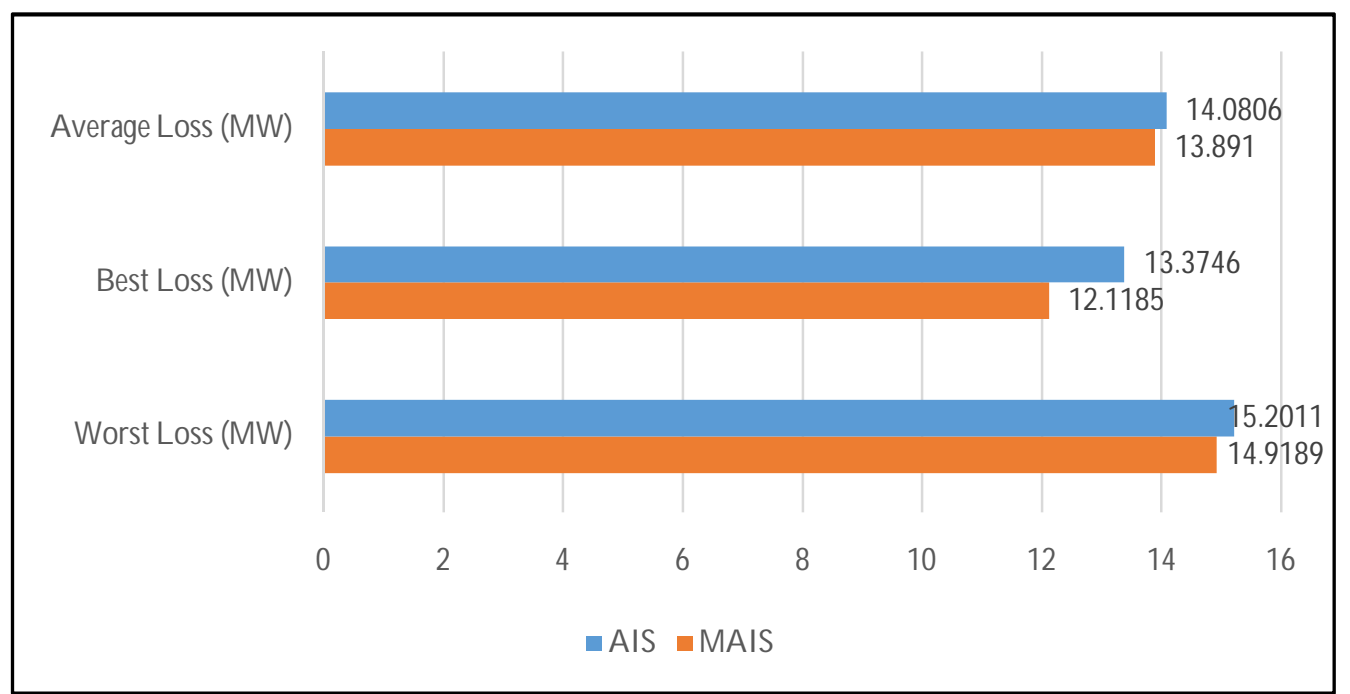

Figure 4: Average Loss, Best Loss and Worst Loss for MAIS and AIS ((N-2) Line Contingency)

\subsection{Case Study 3: (N-2) Line Contingency}

Figure 4 shows the comparison of total system losses found by MAIS and AIS for (N-2) line contingency. Based on Figure 4, the best loss found by MAIS is significantly lower as compared to AIS. The best loss obtained by MAIS and AIS program are $12.1185 \mathrm{MW}$ and $13.3746 \mathrm{MW}$, respectively. This brings to a reduction in percentage difference of about $9.39 \%$ between the two techniques. The same pattern can be seen in the worst loss. The decrement in the worst loss in 
MAIS is $1.86 \%$ as compared to the AIS. However, the worst loss for both techniques are still within the acceptable limits of the $(\mathrm{N}-2)$ line contingency state.

Finally, the average loss for all the 10 runs done using MAIS also shows a decrease in the total system loss as compared to 10 runs done using AIS. The decrement in the average loss is about $1.35 \%$. This indicates that the MAIS is better at finding the least loss for two lines on outage in transmission network.

\section{CONCLUSION}

In a nutshell, the Multistage Artificial Immune System (MAIS) algorithm for the installation of DG in the transmission network has been successfully designed and well- developed using MATLAB programming language to meet the objectives of the project. The developed MAIS algorithm was successfully tested with three different case studies which are Base Case, (N-1) line contingency and $(\mathrm{N}-2)$ line contingency and the results obtained were analyzed thoroughly. There are three different DG units with different sizes optimally placed onto the IEEE 26- Bus RTS during the execution of the case studies. Based on comparison of total system loss produced between MAIS and AIS, it can be concluded that MAIS is superior than AIS to be used for DG placement in transmission network for total system loss minimization.

\section{ACKNOWLEDGEMENT}

The authors would like to acknowledge the Universiti Tenaga Nasional (UNITEN) for the financial support of this project. This research is supported by UNITEN under the BOLD Research Grant 2020 with project code: UNITEN/RMC(BOLD)/1/14/AL/2020/59.

\section{REFERENCES}

1. G. B. Gharehpetian, S. M. Mousavi Agah, and G. B. Gharehpetian.Distributed generation systems : design, operation and grid integration, Oxford, United Kingdom; Cambridge, MA. : Butterworth-Heinemann, an imprint of Elsevier, 2017. (@2017, 2017.

2. H. Zhan et al.Relay protection coordination integrated optimal placement and sizing of distributed generation sources in distribution networks, IEEE Trans. Smart Grid, Vol. 7, no. 1, pp. 55-65, 2016.

3. B. R. Pereira, G. R. M. Martins Da Costa, J. Contreras, and J. R. S. Mantovani. Optimal Distributed Generation and Reactive Power Allocation in Electrical Distribution Systems,IEEE Trans. Sustain. Energy, Vol. 7, no. 3, pp. 975-984, 2016.

4. H. Falaghi and M. R. Haghifam. ACO based algorithm for distributed generation sources allocation and sizing in distribution systems,2007 IEEE Lausanne POWERTECH, Proc., pp. 555-560, 2007.
5. M. Lalitha, N. Reddy, and V. Reddy. Optimal DG placement for maximum loss reduction in radial distribution system using $\mathrm{ABC}$ algorithm,Int. J. Rev. Comput., Vol. 3, pp. 44-52, 2010.

6. R. S. Rao, K. Ravindra, K. Satish, and S. V. L. Narasimham.Power loss minimization in distribution system using network reconfiguration in the presence of distributed generation,IEEE Trans. Power Syst., Vol. 28, no. 1, pp. 317-325, 2013.

7. M. H. Mansor, I. Musirin, M. M. Othman, S. A. Shaaya, and S. A. S. Mustaffa. Application of Immune Log-Normal Evolutionary Programming in Distributed Generation Installation, Indones. J. Electr. Eng. Comput. Sci., vol. 6, no. 3, pp. 730-736, 2017.

8. L. W. De Oliveira, E. J. De Oliveira, F. V. Gomes, I. C. Silva, A. L. M. Marcato, and P. V. C. Resende,Artificial Immune Systems applied to the reconfiguration of electrical power distribution networks for energy loss minimization,Int. J. Electr. Power Energy Syst., Vol. 56, pp. 64-74, 2014.

9. N. S. Razali, N. Roslan, M. H. Mansor, I. Musirin, S. A. Shaaya, and S. Jelani.Optimal Reactive Power Control Using Compensating Capacitor Based on Artificial Immune System, Int. J. Adv. Trends Comput. Sci. Eng., Vol. 8, no. 1.3, pp. 381-386, 2019. https://doi.org/10.30534/ijatcse/2019/6781.32019

10. B. S. Kumar, M. Suryakalavathi, and G. V. N. Kumar. Optimal Power Flow with Static VAR Compensator Using Galaxy Based Search Algorithm to Minimize Real Power Losses, Procedia Comput. Sci., vol. 92, pp. 42-47, 2016. 